\title{
Non-Destructive Testing by Means of Frequency Modulated Infrared Imaging
}

\author{
Kante Murali, Ravibabu Mulaveesala, and D. V. Rama Koti Reddy
}

\begin{abstract}
Infrared non-destructive evaluation (IRNDE) is an emerging approach for non-contact inspection of various solid materials such as metals, composites and semiconductors for industrial and research interest. This paper describes the characterization of possible subsurface failures inside Mild steel materials, which are widely used, particularly in the power and steel industries by Frequency Modulated Thermal Wave Imaging(FMTWI). Mild steel specimens with a man made defects were tested by of recently proposed pulse compression based approach. Present work highlights comparative results obtained with both phase and correlation based approaches for defect detection using frequency modulated thermal excitation scheme.
\end{abstract}

Index Terms-Pulse thermography, Lockin thermography, frequency modulated thermal wave imaging, pulse compression and Phase analysis

\section{INTRODUCTION}

Infrared non-destructive evaluation (IRNDE) is an emerging approach aimed to the detection of surface temperature variations related to presence of a defects in various solid materials such as metals, composites and semiconductors for industrial and research interest.Infrared thermography has become a more popular nondestructive inspection method to evaluate subsurface defects in metallic, insulating, and composite materials, because of its fast inspection rate, noncontact, portability, and easy to interpret. Thermographic methods broadly divided into two types: passive, in which the temperature pattern of thesurface of material is measured without any external thermal excitation, and active in which thermal energy is artificially injected in to the material to be tested [1] . Although several methods have been proposed over the years to detect delamination defects in solid materials, three of them are predominantly in use: Pulse Thermography (PT) lock in Thermography (LT) and Pulse Phase Thermography (PPT) [2],[3],[4]. However, none has so far been free from certain limitations: Pulse thermography(PT) requires high peak power heat sources and being sensitive to surface emissivity variations and nonuniform heating on the surface of test sample, lock in Thermography (LT) suffers with

Manuscript received March 10, 2013; revised May 20, 2013.

Kante Murali is with the Narayana Engineering College, Muthukur road, Nellore:524004, A.P india. (email: Murali.kante@gmail.com)

Ravibabu Mulaveesala is with the Indian Institute of Technology Ropar, Nangal Road, Rupnagar, Punjab, INDIA 140001.(email: ravibabucareiitd@gmail.com)

D. V. Rama Koti Reddy is with the Department of Instrument Technology, Andhra University College of Engineering, Andhra University, Vishakaptanam, Andhra Pradesh, India.(email: rkreddy67@yahoo.co.in) limited depth resolution and long processing time[5],[6] and Pulse Phase Thermography (PPT) needs high peak power heat sources to detect deeper subsurface defects, which however may damage the surface of the test sample. Being prevalent in RADAR, pulse compression methodology allows more energy imposition with low power, long duration stimulation and resolving the detail using compressed profiles [7]-[9] Which can subsequently enhances signal to noise ratio, provides better resolution and facilitates deeper depth probing as well. This paper highlights the defect detection capability of pulse compression method and its edge over conventional phase based detection for FMTWI, with the experimentation carried over a mild steel specimen containing flat bottom holes.

\section{THEORY}

In FMTWI, a linear frequency modulated heat flux is imposed on the surface of the test object. The absorbed heat energy propagates through the object by conduction and produces a time varying thermal response over the surface of the object depending on the homogeneity of the substance. The theoretical model to study this thermal response is based on $1 \mathrm{D}$ heat equation

$$
\frac{\partial^{2} \mathrm{~T}(x, t)}{\partial^{2} \mathrm{x}}-\frac{1}{\alpha} \frac{\partial \mathrm{T}(x, t)}{t}=0
$$

where $\alpha$ is the thermal diffusion coefficient, $T(x, t)$ is the instantaneous temperature on the surface and $\mathrm{x}$ is the direction of heat flow (perpendicular to the surface). On solving the above equation for FMTWI under boundary conditions (21), instantaneous temperature is obtained as

$$
\begin{aligned}
& T(x, t)=T_{0} \exp \left[-x \sqrt{\frac{\pi\left(f_{0}+b t\right)}{\alpha}}-j x \sqrt{\frac{\pi\left(f_{0}+b t\right)}{\alpha}}\right] \\
& \exp \left[2 \pi j\left(f_{0} t+(b / 2) t^{2}\right)\right]
\end{aligned}
$$

where $B / \tau$ is the frequency sweep rate of the chirp and $\tau$ is duration of excitation.

The thermal diffusion length of this frequency modulated thermal wave is given by

$$
\mu=\sqrt{\frac{\alpha}{\pi\left(f_{0}+\frac{b t}{\tau}\right)}}
$$


The dependence of thermal diffusion length (Equation 3) on the bandwidth of the modulated excitation assures the depth scanning of the sample, with the suitable band of frequencies in a Single frequency modulation cycle.

\section{MATERIALS AND EXPERIMENTATION}

Experimentation has been carried over a mild steel sample of dimensions as shown in Fig. 1. (a). The experimental mild steel sample contains six drilled bottom holes of diameter $1 \mathrm{~cm}$ each kept at different depths from the non defective end. A frequency modulated chirped stimulation of frequencies swept from $0.01 \mathrm{~Hz}$ to $0.1 \mathrm{~Hz}$ in $100 \mathrm{~s}$, has been provided to the sample with the help of two halogen lamps of power $1 \mathrm{~kW}$ each. Lamps are driven by a built in control unit as shown in the experimental set up of Fig. 1. (b) with the chosen stimulation. Temporal thermal response has been captured at a frame rate of $20 \mathrm{~Hz}$ using a mid band infrared camera. A suitable processing method is to be applied over the captured thermogram sequence and subsurface information is extracted.

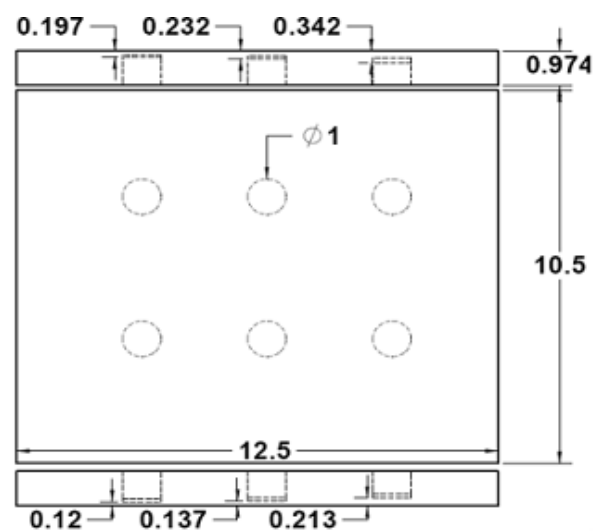

(a)

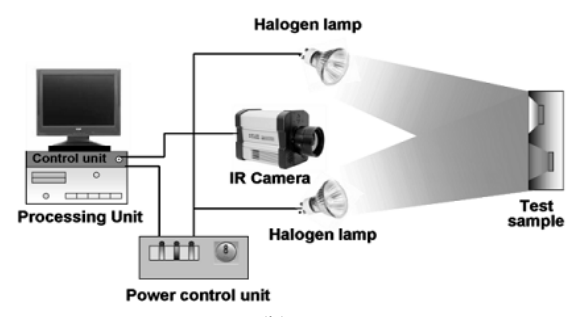

(b)

Fig. 1.(a). Layout of the experimental mild steel sample (b). Experimental set up

\section{RESUlTS AND DisCUSSION}

In order to reveal subsurface features, the offset in temporal thermal profiles of each pixel is removed by a linear fit and further processing has been carried over these mean removed profiles of each pixel in view. Fig. 2.a shows the mean removed temporal thermal profile of a non defective pixel contaminated with experimental noise. In general thermal responses from deeper subsurface anomalies are more contaminated by noise and produces low signal to noise ratio (SNR) as shown in Fig. 2.b. This results in the deeper anomalies to be buried in noise and provide false alarms in detection. Processing methods applied over these profiles to reduce noise influence or improving signal to noise ratio of these profiles will enhance detection performance and reveal deeper subsurface features.

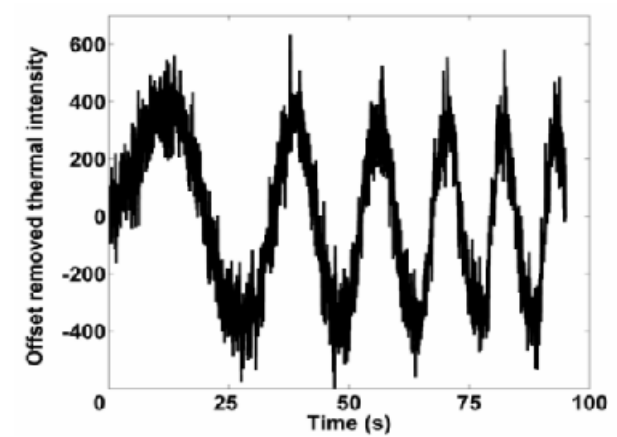

(a)

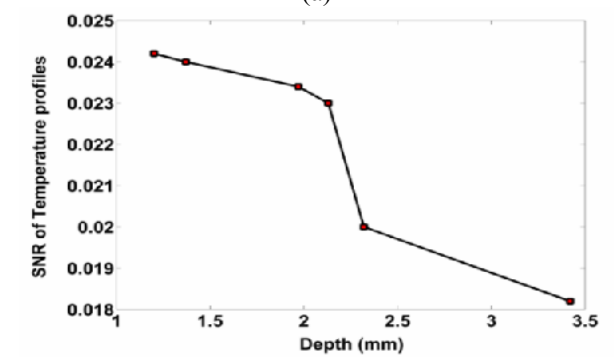

(b)

Fig. 2 a. Temporal thermal profile of a non defective pixel b. SNR of profiles vs. depth of the defect

Pulse compression applied over these noise contaminated profiles enhances the SNR and provides better defect detection for coded excitations, which has been adopted in this contribution in addition to conventional phase based methodology for defect detection. In correlation based pulse compression, mean removed thermal profiles of each pixel has been cross correlated with a reference profile and correlation coefficient contrast due to depth dependent delay is used for detection. Let $s(t)$ the actual response and $n(t)$ be the noise contaminated with the profile and $r(t)$ is the reference. Cross correlation of the profiles is given by

$$
\begin{aligned}
y(t) & =\int(s(\tau)+n(\tau)) r(t+\tau) d \tau \\
& =(s(t)+n(t)) \oplus r(t) \\
& =(s(t) \oplus r(t)+n(t) \oplus r(t)) \\
& =s(t) \oplus r(t)
\end{aligned}
$$

As random noise $n(t)$ may not correlate with $\mathrm{r}(\mathrm{t})$ results in the second terms of equation 4 to zero. Pulse compression results in an improved SNR of $10 \log (\tau B)$. Where ' $\tau$ ' is the experimentation time and ' $\mathrm{B}$ ' is the band width. Where as conventional phase analysis results in a phase profile of variance

$$
\sigma_{s}=\left(1 / E^{2}\right) \sigma_{n}
$$

where $\sigma_{n}$ the variance of the noise and $E$ is the energy of 
the profile. Thus the phase response depends on the noise parameters, where as pulse compression minimizes it.

On the application of the above processing methods over mean removed temporal profiles, correlation image can be developed by arranging the normalized correlation coefficients at delayed instants in appropriate pixel position. Correlation coefficient contrast depending on the delay is used to identify defect signatures in this approach. Where as in phase analysis, FFT is applied over the temporal profiles of each pixel and phase information is obtained by taking the

$$
\phi(n)=\tan ^{-1}\left(\frac{\operatorname{Im}\left(S_{n}\right)}{\operatorname{Real}\left(S_{n}\right)}\right)
$$

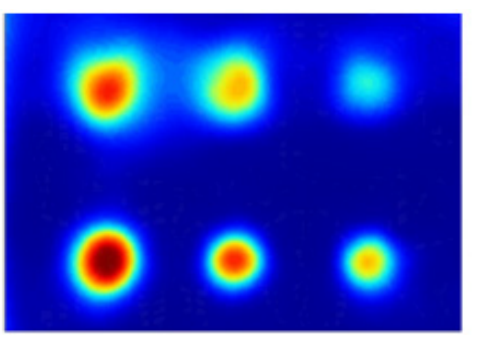

(a)

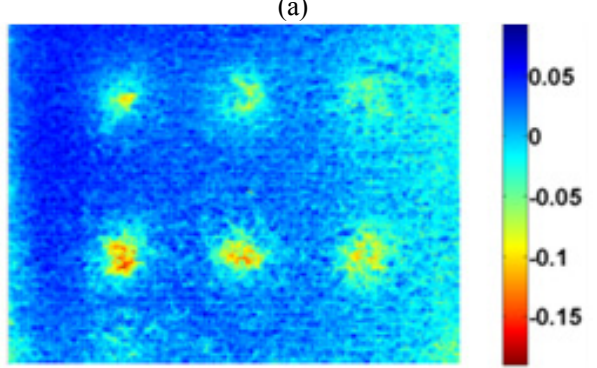

(b)

Fig. 3. (a). Pulse compression at delayed instant of $15.2 \mathrm{~s}$ (b). Phase image at $0.02 \mathrm{~Hz}$

where $S_{n}$ is the $n^{\text {th }}$ term in the FFT of the profile. Phase contrast has been computed by subtracting the Phase contrast in a phasegram is used to individuate defects. Frequency of the phasegram is obtained from $F_{n}=n \frac{F_{s}}{N}$ where ' $n$ ' is number of the phasegram.

Correlation image at $15.2 \mathrm{~s}$ as shown in Fig. 3(a) visualizes all the defects clearly than the phase image obtained at $0.02 \mathrm{~Hz}$ as shown in Fig. 3. b. Enhanced detection capability in pulse compression under noisy environment has been clearly represented in this context. In thermal wave propagation through the material over the defects at different depths, waves can not only attenuated but also delayed. This delay is a measure of depth of the defect which can be assessed using peak delays in pulse compression. Delay in correlation peaks represents the group delay between the temporal profiles of the defect location with the reference profile. Fig. 4 (a) illustrates the peak delay of the correlation profiles corresponding to the centers of the defects. The shallowest defect with its most delayed profile (as compared to reference) is far from that of the deepest one as the non defective profile has been considered as reference (enlarged view of peaks is shown in insert of Fig. 4. (a). Peak delay vs depth of the defect has been empirically fitted as shown in Fig. 4. (b). In order the asses the detectability of defect detection, the SNR of pulse compression and Conventional FMTWI were calculated and compared as shown in Table I. Pulse compression approach exhibits better detectability than the remaining schemes.
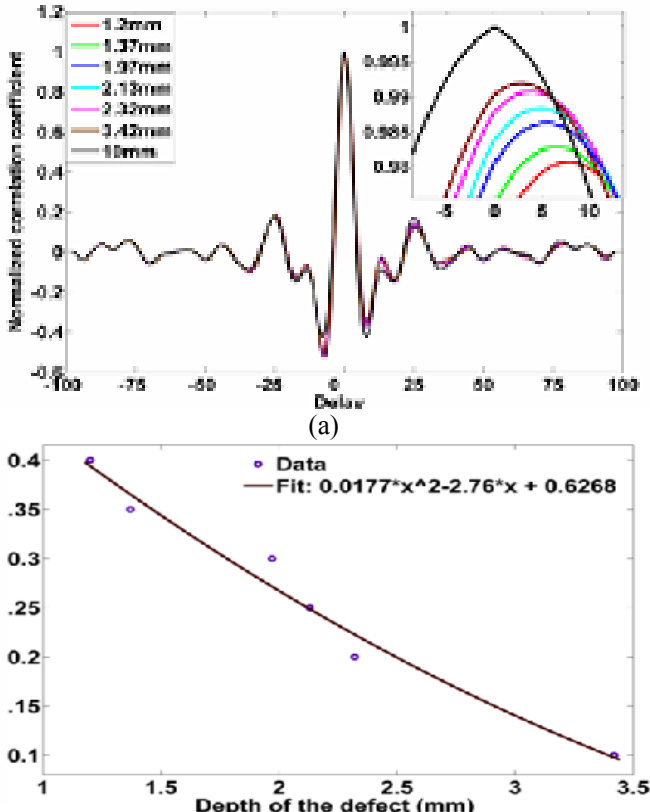

(b)

Fig. 4. (a). Pulse compression profiles of pixel of defect centers (b). Peak delay vs depth

TABLE I: PULSE COMPRESSION APPROACH EXHIBITS BETTER DETESTABILITY THAN THE REMAINING SCHEMES

\begin{tabular}{|c|l|l|l|l|l|l|l|}
\hline Approach & \multicolumn{7}{|c|}{ SNR Values in $\mathbf{d B}$} \\
\hline $\begin{array}{c}\text { Depth ----> } \\
(\mathbf{m m})\end{array}$ & $\mathbf{1 . 2}$ & $\mathbf{1 . 3 7}$ & $\mathbf{1 . 9 7}$ & $\mathbf{2 . 1 3}$ & $\mathbf{2 . 3 2}$ & $\mathbf{3 . 4 2}$ & $\mathbf{1 0}$ \\
\hline FMTWI Phase & 86 & 84 & 80 & 78 & 74 & 65 & 40 \\
\hline $\begin{array}{c}\text { FMWTI Pulse } \\
\text { compression }\end{array}$ & 91 & 87 & 83 & 81 & 78 & 72 & 45 \\
\hline
\end{tabular}

\section{CONCLUSIONS}

Defect detection capability of frequency modulated thermal wave imaging with recently introduced pulse compression method has been emphasized and compared with the conventional phase based analysis. Pulse compression with its SNR improvement enhanced the defect detection capability and provides the subsurface details with more effectively than phase based methodology. It has been clearly visualized with the deepest defect located at the top right end of the specimen.

\section{REFERENCES}

[1] X. Maldague, S. Marinetti, "Pulse Phase Infrared Thermography," J. Appl. Phys, vol. 79, no. 5, pp. 2694-2698, 1996.

[2] S. M. Shepard, "Advances in Pulsed Thermography," in Proc. of SPIE, vol. 4360, pp. 511-16, 2001.

[3] D. Wu and C. Busse, "Lock -in thermography for Nondestructive evaluation of materials," Rev. Gin. Therm, vol. 37, pp. 693-98, 1998.

[4] X. Maldaguea, F. Galmichea, and A. Ziadia, "Advances in Pulsed Phase Thermography," in Proc. SPIE, vol. 1 no. 1, 2001.

[5] R. Mulaveesala, P. Pal, and S. Tuli, "Interface study of bonded wafers by digitized linear frequency modulated thermal wave imaging," Sens. Actuators A., vol. 128, pp. 209-214, 2006,

[6] R. Mulaveesala and S. Tuli, "Theory of frequency modulated thermal wave imaging for nondestructive sub-surface defect detection," Appl. Phys. Lett, vol. 89, pp. 1913-1918, 2006.

[7] R. Mulaveesala, J. S. Vaddi, and P. Singh, "Pulse compression approach to infrared non-destructive characterization," in Proc. Review of Scientific Instruments, vol. 79, no. 9, pp. 094901, 2008 
[8] V. S. Ghali, N. Jonnalagadda, and R. Mulaveesala, "ThreeDimensional Pulse Compression for Infrared Nondestructive Testing," IEEE Sensors Journal, vol. 9, no. 7, pp. 832-33, 2009.

[9] V. S. Ghali, R Mulaveesala, and M Takei, "Frequency-modulated thermal wave imaging for non-destructive testing of carbon fiberreinforced plastic materials," Meas. Sci. Technol, vol. 22, pp. 104018104022, 2011

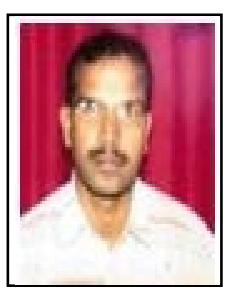

Kante Muralil was born in Nellore, Andhra Pradesh, India. He received AMIETE (B. Tech) degree in electronics and telecommunication engineering From IETE, New Delhi. He did his M. Tech. in materials science in December 2004 from Indian Institute of Technology(IIT), Kanpur. He is currently a Ph.D. student at A. U. College of Engineering, Andhra University, Visakhapatnam, India.

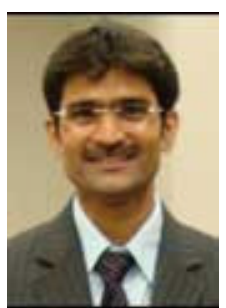

Ravibabu Mulaveesala was born in Chukkavanipalem, A. P., India. He received B.Sc. (mathematics, physics and electronics) from M.R. College, M.Sc. in Electronics from Nagarjuna University and M. Tech. in non-destructive testing from national institute of technology (NIT), Tiruchirappalli and a Ph.D. from Indian Institute of Technology(IIT) Delhi, India. He is presently working as a Assistant professor in Department of
E.C.E, IIIT, Jabalpur, india. $\mathrm{He}$ is involved in non-destructive characterization of materials specially using thermal wave imaging

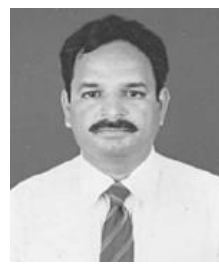

D. V. Rama Koti Reddy received the $\mathrm{Ph}$. D. degree from Andhra University, Visakhapatnam, India, in 1996. He is presently working as an Associate Professor with the Department of Instrumentation Engineering, Andhra University. He has more than 20 publications in peer reviewed international journals, national journals, and book chapters. His teaching and research interests are biomedical signal adaptive signal processing. processing, wireless sensor networks, MEMS, and 